\title{
On the Relation between Chemical Composition and Optical Properties of Detonation Nanodiamonds
}

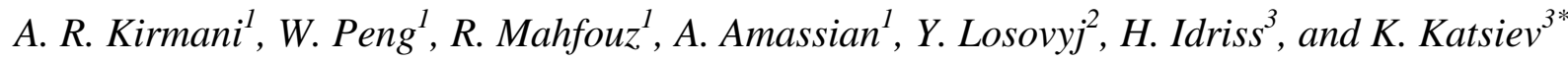

${ }^{1}$ King Abdullah University of Science and Technology (KAUST), Physical Sciences and Engineering Division, Solar and Photovoltaics Engineering Research Center (SPERC), Thuwal, 23955-6900, Saudi Arabia

${ }^{2}$ Department of Chemistry, Indiana University, Bloomington, IN 47405, USA

${ }^{3}$ SABIC Corporate Research and Innovation Center, King Abdullah University of Science and Technology (KAUST), Thuwal, 23955-6900, Saudi Arabia

*: Corresponding author. Tel: +966 542-585885. E-mail: katsievk@ SABIC.com 
The morphology and presence of impurities strongly influence mechanical, optical, electrical, and thermal properties of detonation nanodiamonds (DNDs). Here we report insights on the chemical composition and its effect on the optical properties of the DNDs obtained by rate-zonal density gradient ultracentrifugation. Herein, for the first time, a detailed valence band structure of as-prepared and oxidized DNDs is reported. Photoemission spectroscopy (PES) measurements demonstrate that the defects, originating from fullerene-like $\mathrm{C}$ bonding in the $\mathrm{sp}^{2}$ shells of the DNDs, are governing the literature-reported loss of the emission spectral features arising from the nitrogen-vacancy (NV) center excitations. X-ray photoelectron spectroscopy (XPS) measurements reveal that nitrogen is present in the DNDs in the form of $\mathrm{N}-\mathrm{O}$ bonded species located at the surface region $/ \mathrm{sp}^{2}$ shells, while in core of the DND it is in the form of $\mathrm{N}-\mathrm{C} / \mathrm{N}=\mathrm{C}$ species.

\section{Introduction}

Detonation nanodiamonds are produced by the detonation of explosives in a closed chamber in an oxygen-deficient atmosphere. They have the smallest particle size among different kinds of synthetic diamonds, typically in the range of $\sim 10 \mathrm{~nm}$, and exhibit outstanding mechanical, optical, electric, and thermal properties.(1) This makes DNDs promising in a diversity of areas of science and technology, ranging from the 'conventional' applications such as abrasives, cutting and polishing tools, $(2,3)$ to the 'cutting-edge' ones - drug delivery, biomedical imaging, non-toxic contrast agents,(4-7) magnetic sensors,(8) composites, $(9,10)$ and quantum computing.(11-13) 
The structure and chemical composition of DNDs strongly influence their mechanical, optical, electrical, and thermal properties.(14-18) One prominent example is the nitrogen doping which plays an important role in the fluorescence of the DNDs.(19) Nitrogen defects are most prominent in diamond since nitrogen is a common impurity of the material. Its concentration in DNDs, the ability to control the doping, and the production of nitrogen-vacancy (NV) centers are of great importance for the fabrication of single color centers. An NV center comprises of a pair of substitutional nitrogen atom and a nearby lattice vacancy in the nanodiamond. These centers exist in two charge states: $\mathrm{NV}^{0}$, a neutral species and $\mathrm{NV}^{-}$, a negatively charged species.(1) Due to their strong florescence, the NV centers are the key component in the DND-based quantum computing applications. The application of color centers in diamond relies on progress in quality control of diamond material and the ability of precise generation of defects.(20-22)

Presence of nitrogen also strongly affects the mechanical properties of the carbon-based materials. Sjostrom et al. reported that $\mathrm{CN}$ films that have fullerene-like microstructure consisting of a three-dimensional network of covalently bonded $\mathrm{C}$ and $\mathrm{N}$ atoms exhibit extreme mechanical properties, with hardness of $60 \mathrm{GPa}$ and an elastic recovery as high as $85 \%$.(23) It has been demonstrated that the addition of $\mathrm{N}$ induces buckling of the basal planes, enabling cross-linking between the planes via sp-hybridized carbon. This cross-linking strengthens the weak bonds between the basal planes and results in a structure having the rigidity of a threedimensional covalently bonded system.

Despite the large number of studies on DNDs and the demonstrated high potential for a variety of applications, fundamental understanding of the structural and chemical composition of the DNDs remains relatively poor. The DNDs are known to comprise of an $\mathrm{sp}^{3}$ carbon diamond core covered with a shell of $\mathrm{sp}^{2}$ (graphitic) carbon. $(24,25)$ Most studies that were performed on the 
DNDs involve the oxidative purification step that considerably changes their shape and properties.(1) The effect of the purification step on the chemical composition of the DNDs however has not yet been studied in detail. The goal of this study is to gain insights into the structural and chemical composition of the $\mathrm{sp}^{2}$ shell and the $\mathrm{sp}^{3}$ core of the diamonds in contrast to the state-of-the-art consideration of the DND as a single photo-luminescent entity. The study aims at answering another important question raised by Mochalin et al., namely, the role of surface chemistry of the DNDs in modifying the brightness of fluorescence from the NV centers.(1) Elucidating the correlation between the electronic structure and optical properties of as-prepared versus oxidized DNDs will aid the fundamental understanding, assure the quality, and make them attractive for basic studies as well as engineering applications.

\section{Experimental}

\subsection{Detonated Nanodiamonds (DNDs) preparation. The DNDs, purchased from Sigma} Aldrich, were prepared by rate-zonal density gradient ultracentrifugation, described elsewhere.(26)

2.2. Oxidation procedure. 2 grams of as-received DNDs (Detonated Nanodiamonds) were uniformly mounted on the bottom of an alumina crucible (MTI Corporation, USA, dimension $100 \times 40$ x $18 \mathrm{~mm}$ ), and then loaded in a muffle furnace (Lindberg Blue M, Thermo Scientific).

The oxidation process was carried out at $425^{\circ} \mathrm{C}$ for 48 hours with an 8 -minutes ramp of heating and cooling process. The oxidized DNDs were then collected and treated with excess hydrochloride acid (37\%, ACS reagent, Sigma-Aldrich) to remove the metal or metal oxide impurities that were previously unreachable due to the shielding of carbon. The mixture was centrifuged and rinsed with MilliQ water for several times. The final stable solution of DNDs was obtained by dispersing the particles in $\mathrm{pH} \sim 10$ solution at a rough concentration of $10 \mathrm{mg} / \mathrm{mL}$; 
water adjusted with ammonium hydroxide (28-30\%, ACS reagent, Sigma-Aldrich), and stored for future fractionation and characterizations.

2.3. Transmission Electron Microscopy (TEM). TEM images were obtained using a $\operatorname{TitanG}^{2} 80$ 300 instrument, equipped with an image-corrector from CEOS. The instrument was equipped with energy-filters from Gatan Inc. (the filters were however not used for the images reported herein). $2 \mu \mathrm{L}$ of DNCs suspended in water was deposited on an ultrathin carboncoated films on 300 mesh copper grids and dried in air for at least $1 \mathrm{~h}$. Detonation nanodiamonds are subjected to re-aggregation during the drying process in preparation of TEM grids. Shenderova et al. suggested that re-aggregation can be largely circumvented by dispersing DNDs in DMSO/Methanol mixture.(46) Here, we dispersed the dried sample powder in DMSO followed by the addition of an equal volume of methanol to the solution. The mixture was then sonicated (sonication bath: Bransonic, Mexico) for one hour, followed by deposition onto the ultrathin TEM grids.

2.4. X-ray Photoelectron Spectroscopy (XPS). The DND samples for XPS were prepared via drop-casting from an aqueous solution onto a high purity (99.9\%) copper (Cu) foil. Measurements were carried out in a UHV chamber with a base pressure of $\sim 2 \times 10^{-10}$ Torr. The chamber was equipped with the variable temperature scanning tunnelling microscope (STM) Omicron VT STM XA 50/500, SPHERA U7 hemispherical energy analyzer with 7 channel MCD detector, high intensity He I/II lamp HIS 13 ultraviolet photon source, XM 1000 monochromated X-ray source: high intensity, high energy resolution monochromated Al Ka Xray source with $500 \mathrm{~mm}$ Rowland circle diameter, minimum spot size $\sim 1 \mathrm{~mm}$, photon line width $<250 \mathrm{meV}$. Spectra were acquired at normal emission using Al $\mathrm{K}_{\alpha}(1486.6 \mathrm{eV})$. The photoelectrons were collected by the hemispherical energy analyzer operated in constant 
analyser energy (CAE) mode.

2.5. Fluorescence measurements. Fluorescence measurements were carried out with a NanoLog (HORIBA Jobin Yvon, USA) configuration in a front-face detection set-up. A 450W Xenon lamp was used as the excitation source. $470 \mathrm{~nm}$ monochromatic light was obtained through a $1200 \mathrm{gr} / \mathrm{mm}$ excitation monochromator and used to excite samples. Emission was captured by a standard iHR320 R928 PMT 250-850 nm detector (Horiba Scientific, USA).

2.6. Raman spectroscopy. UV Raman spectra were captured with Aramis Raman Spectroscopy (Horiba Scientific, USA) by loading dried samples (dried under vacuum at room temperature in a Thermo Scientific vacuum oven) on a special sample holder (Linkam 600) with argon flow and using a $325 \mathrm{~nm}$ UV laser. The capturing parameters was 15 seconds for 4 cycles, to minimize the laser burning-off effects but maintain a high enough signal to noise ratio at the same time.

2.7. Photoemission spectroscopy. The ultraviolet photoemission spectroscopy measurements were carried out in PHI Versa Probe II instrument UHV chamber equipped with He ultraviolet discharge source. The He II radiation was used for all experiments. Photoemission spectra were recorded using PHI software SmartSoft -XPS v2.0 with the pass of $2.9 \mathrm{eV}$, energy step of 25 meV and processed using PHI MultiPack v9.0.

\section{Results and Discussion}

Chemical composition and sample cleanliness of as-prepared DNDs, prepared by the established procedure, (26) was studied with XPS (Fig. S1) and showed that the DNDs mainly comprise of $\mathrm{C}$, with small amounts of $\mathrm{O}, \mathrm{N}$, and traces of $\mathrm{Cl}$. The presence of $\mathrm{Cl}$ is explained by the $\mathrm{HCl}$ acid treatment. The detected oxygen is commonly observed in the samples that have been exposed to air, if no further treatment e.g. degassing, plasma or similar treatment was done, 
prior to transfer to the ultra-high vacuum (UHV) chamber. This is explained to be due to water molecules or/and surface hydroxyls adsorbed on the surface.

Oxidative purification procedure is commonly utilized in the DNDs research studies and has proven to be an effective method of removing the $\mathrm{sp}^{2}$ shell. Thus, in order to gain an understanding of the role of the $\mathrm{sp}^{2}$ shell, a comparative study of the electronic structure and chemical composition of DNDs before and after the removal of the $\mathrm{sp}^{2}$ shell was performed.

Transmission electron microscopy (TEM) performed on the DNDs showed that they consist of agglomerates of several crystalline primary particles embedded in an amorphous matrix as shown in Fig. 1 (A and B), consistent with the other TEM reports on DNDs.(24) Occasionally, single-crystalline particles are also observed (not shown). The as-prepared DNDs are surrounded by layers of $\mathrm{sp}^{2}$ shell (Fig. 1(B)) that gets significantly reduced after the oxidative purification procedure (Fig. 1(D)), while a certain degree of agglomeration remains (Fig. 1(C)). The average particle size of as-prepared DNDs was estimated to be $\sim 100 \mathrm{~nm}$, and was found to reduce to $35 \mathrm{~nm}$ following oxidation (without measuring the involved DND dispersion and the agglomeration). The overall removal of the $\mathrm{sp}^{2}$ shell was monitored by Raman spectroscopy and revealed its substantial reduction (see Supplementary Fig. S2) consistent with the TEM measurements. The as-prepared sample shows an intense, broad peak around $1600 \mathrm{~cm}^{-1}$ corresponding to the G-band of graphitic carbon (red spectrum, Fig. S2). The diamond peak can be observed as a weak shoulder near $1330 \mathrm{~cm}^{-1}$. However, upon oxidation this grows into a sharp spectral feature (blue spectrum, Fig. S2) signaling significant removal of the $\mathrm{sp}^{2}$ shell. $(28,29)$ 

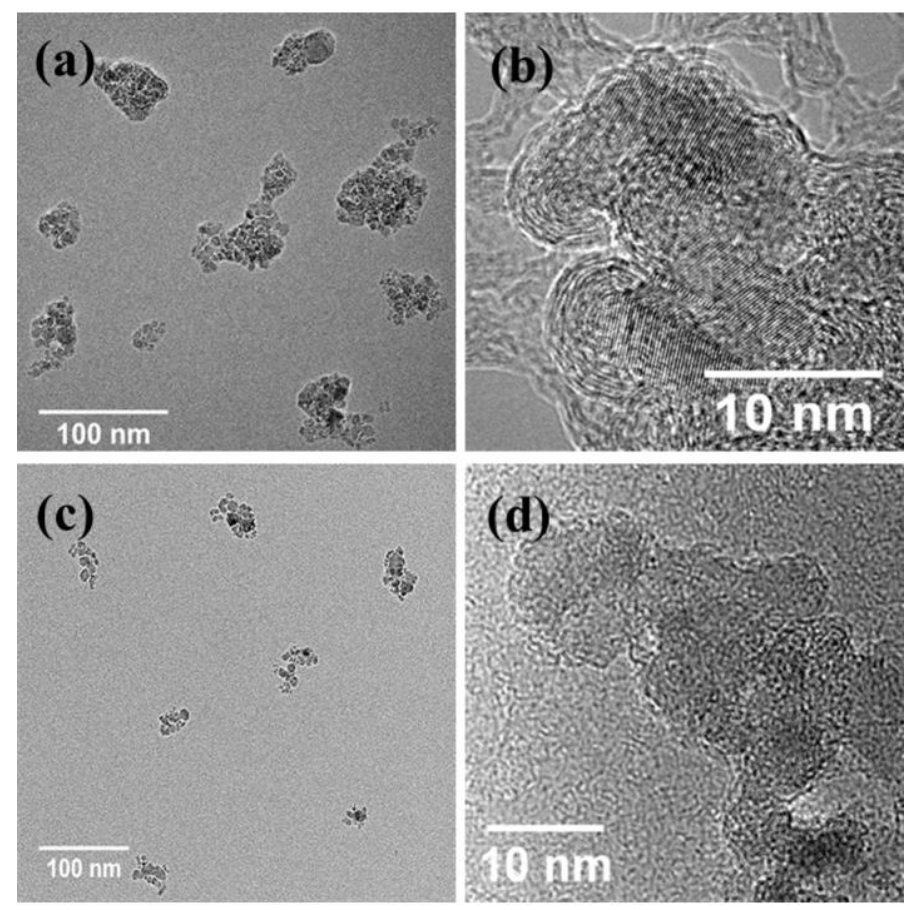

\section{Figure 1.}

TEM of DND-agglomerates consisting of several crystalline primary particles (A), embedded in the amorphous carbon matrix (B). The $\mathrm{sp}^{2}$ shell seen in $(\mathrm{B})$ is significantly reduced after the oxidative purification procedure (D), while certain degree of agglomeration remains (C).

Surface properties have been shown to play a defining role in determining the physicalchemical properties of small DNDs $(\sim 10-20 \mathrm{~nm})$ owing to their large surface-to-volume ratio.(27) Prior to the removal of $\mathrm{sp}^{2}$ shell through oxidation, DNDs exhibit a broad featureless photoluminescence (PL) spectrum. This has been linked to the presence of defects in the graphitic shell.(27-29) However, a direct evidence of such defects has not yet been reported. Figure 2 compares the fluorescence spectrum of the DNDs covered with graphitic shells with that of the oxidized DNDs. As expected, oxidation of the shell leads to development of prominent features in the emission spectra. The features observed after the shell removal correspond to $\mathrm{NV}^{0}$ and $\mathrm{NV}^{-}$centers. $(28,30)$ 


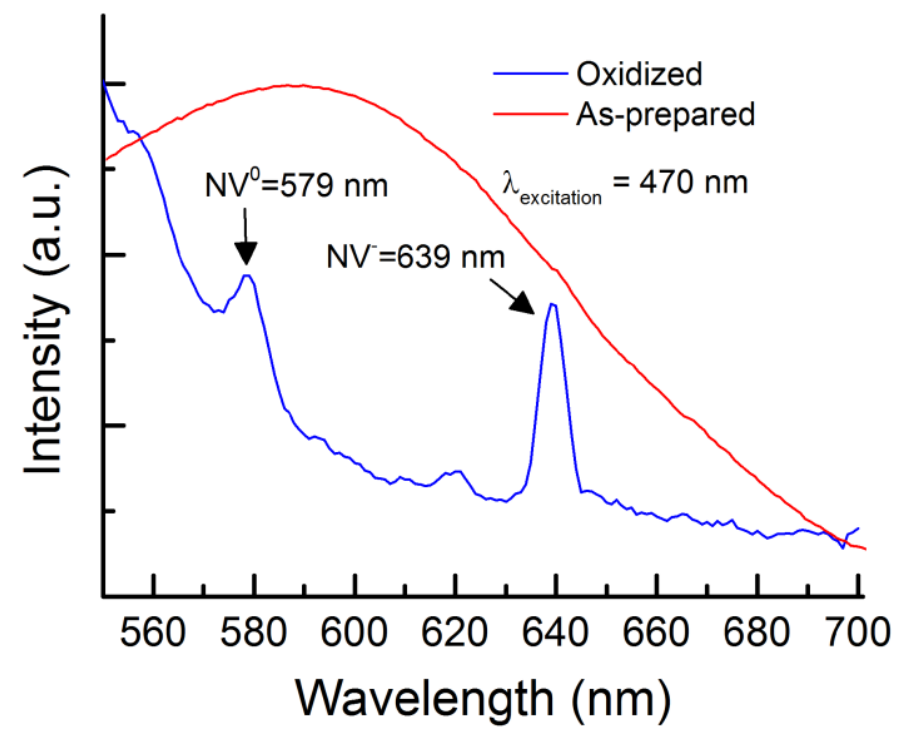

\section{Figure 2.}

Fluorescence spectra showing development of strong emission features near $579 \mathrm{~nm}\left(\mathrm{NV}^{0}\right)$ and $639 \mathrm{~nm}\left(\mathrm{NV}^{-}\right)$for the oxidized samples. The non-oxidized sample shows a broad, featureless emission in the same region.

In stark contrast, the non-oxidized diamonds show a broad, background-like emission. To gain a better understanding of the effect of the graphitic shell on the emission we compared the shellcovered and oxidized DNDs using photoemission spectroscopy (PES) which allows direct probing of the electronic structure. We have deployed similar methodology in the recently reported PES studies of trap states in lead sulfide $(\mathrm{PbS})$ colloidal quantum dot (CQD) based solar cells. $(31,32)$

We report, for the first time, detailed valence band structure of as-prepared and oxidized DNDs. The spectra closely resemble the band structure reported for amorphous $\mathrm{C}$ and $\mathrm{CN}$ films.(33-37) Figure 3(c) shows the valence bands of the shell-covered and oxidized DNDs. The spectra were referenced to the $\mathrm{sp}^{2}$ components of the $\mathrm{C}(1 \mathrm{~s})$ core level peaks, Figures 3(a) and 3(b), which were charge-neutralized at $284.8 \mathrm{eV}$. 

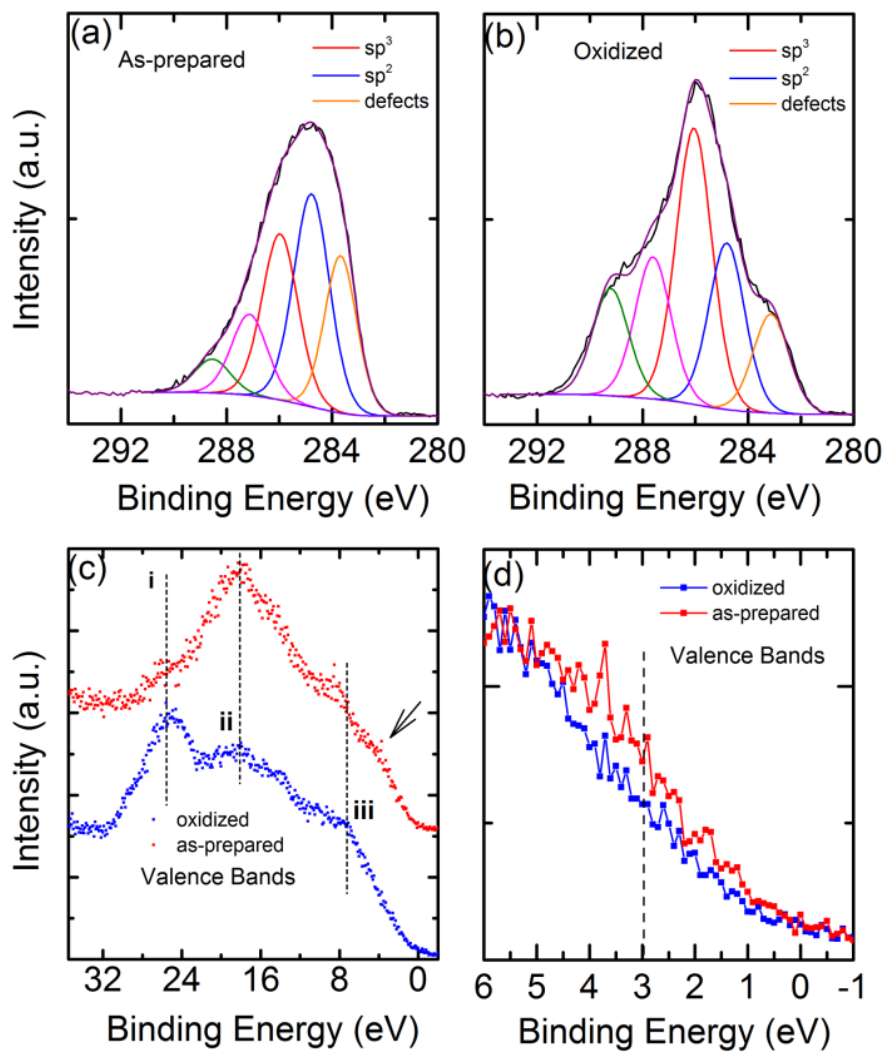

Figure 3.

C1s core level peaks for the (a) as-prepared and (b) oxidized DNDs are shown. (c) Valence bands of the as-prepared and oxidized DNDs. Graphitic shell covered DNDs have an enhanced DOS near $3.0 \mathrm{eV}$ below the Fermi level (black, dashed line) as highlighted in the zoomed spectrum in $(d)$.

The VB spectra show three prominent features (i, ii, iii). (i) and (iii) appear centered around 7 $\mathrm{eV}$ and $24 \mathrm{eV}$, respectively, while (ii) is a broader peak extending from $11 \mathrm{eV}$ to $20 \mathrm{eV}$. O 2p and 2 s electrons give rise to features near $7 \mathrm{eV}$ and $24 \mathrm{eV}$, respectively for the oxidized sample (blue spectrum). The split in the band stretching from $11 \mathrm{eV}$ to $20 \mathrm{eV}$ has been reported for 
graphite, diamond and amorphous carbon films and is suggested to be arising from the s-states of carbon with the split representing an absence of odd-membered rings. $(36,37)$

The VB of the as-prepared DNDs shows the s-band from $11 \mathrm{eV}$ to $20 \mathrm{eV}$ without a split (red spectrum). This hints towards the presence of odd-membered rings in the graphite shell.(37) The enhancement near $8 \mathrm{eV}$ is from $\sigma$ bonds of $\mathrm{C} 2 \mathrm{p} .(33,38)$ Figure $3(\mathrm{~d})$ shows a close-up of the near-Fermi level region for the two samples. There is an enhancement of the density of states (DOS) in 3-4 eV region of the VB for the as-prepared DNDs (indicated by the blue arrow in Fig. 3(c)). The studies of amorphous CN films have demonstrated that an enhanced DOS near the Fermi level is due to lone pair of electrons from $\mathrm{N}$ populating the conduction band of $\mathrm{sp}^{2}$ carbon thereby creating defects.(33) Also, for amorphous $\mathrm{C}$ films, $\pi$ bonds of $\mathrm{C} 2 \mathrm{p}$ have been reported to contribute to the feature around $3-4 \mathrm{eV} .(34,36,37)$ The enhanced density near the Fermi level was therefore attributed to contributions from C $2 \mathrm{p} \pi$ bonds and defect states in the $s p^{2}$ carbon shell owing to formation of 5-membered carbon atom rings,(33): the $\mathrm{N}$-induced fullerene-like defects as a result of buckling of graphitic carbon sheets are common in $\mathrm{CN}$ films.(33, 35) This is consistent with the suppression of the $283.7 \mathrm{eV}$ component of $\mathrm{C} 1 \mathrm{~s}$ core level peak observed upon oxidation (Fig. 3(b)). This component is attributed to the 5-membered carbon ring defects present in the graphitic shell of the DNDs. $(39,40)$

We suggest that these defect states, originating from the fullerene-like bound $\mathrm{C}$ species in the $\mathrm{sp}^{2}$ shells on the as-prepared DNDs, serve as traps for the photoexcited charge carriers, responsible for the loss of the $\mathrm{NV}^{-}$and $\mathrm{NV}^{0}$-related emission spectral features, (tentatively) via radiative recombination channels.(41)

It is worth mentioning that the oxidation method, although efficient in removing the $\mathrm{sp}^{2}$ shell, introduces oxygen into the $\mathrm{sp}^{3}$ cores. The exact mechanism of the oxygen introduction i.e. either 
substitution or interstitial incorporation is unknown and is beyond the scope of the current study. Figure S3 shows the oxygen 1s core level peaks for the as-prepared and oxidized samples and the relative atomic concentrations as measured by XPS are summarized in Table SI1. Oxygen content is found to increase upon oxidation.

We have found that for the as-prepared DNDs, oxygen resides mostly in the graphitic shell and decreases as the shell is removed by controlled annealing in UHV conditions (see Table SI2). However oxidized DNDs were not found to lose their oxygen content upon annealing even at elevated temperatures (above $500^{\circ} \mathrm{C}$ ), indicating that oxygen atoms were incorporated into the $\mathrm{sp}^{3}$ cores of the DNDs. The post-oxidation penetration of oxygen into the core might lead to an interaction of the NV centers with the oxygen atoms.

XPS study revealed that the N1s spectra exhibit two pronounced spectral features, as shown in Fig 4a (N1 and N2 for the lower and higher BE components, respectively), that are clearly resolved for all spectra, and are centered around the $\mathrm{BE}$ positions $399.0 \mathrm{eV}$ and $402.5 \mathrm{eV}$ for the as-prepared sample. The lower BE component was further split into three components, each with a FWHM of $1.4 \mathrm{eV}$. Due to varying and system-dependent reports available in the literature for the $\mathrm{N}$ 1s peak components it is difficult to make an unambiguous peak assignment. However considering that the shift in the N1s from higher to lower binding energy (BE) tracks the degree of hybridization $\left(\mathrm{BE}(+/-0.2 \mathrm{eV})\right.$ of $\left.-\mathrm{NH}_{3}{ }^{+} 401.5>-\mathrm{NH}_{2} 400>=\mathrm{NH} 398.5>\equiv \mathrm{N} 397 \mathrm{eV}\right)$, we assign the three components of the $\mathrm{N} 1$ peak to three different $\mathrm{N}$ containing species with main contribution (the largest component) corresponding to an $\mathrm{sp}^{3}$ such as in $\mathrm{C}-\mathrm{NH}_{2}$ species. $(42, \mathbf{4 3})$ It is also clear that no $-\mathrm{C} \equiv \mathrm{N}$ species are present. The second feature centered around $402.5 \mathrm{eV}$ is typically assigned to $\mathrm{N}-\mathrm{O}$ bonds.(44) This is in agreement with the recent report by Zhu et al. where the authors observe two main spectral features associated with the $\mathrm{N}$ 1s core level peak; 
the higher BE feature has been linked to oxidized pyridinic $\mathrm{N}$ while the feature at lower BE is suggested to be a contribution of pyridinic, pyrrolic and $\mathrm{sp}^{3}$-bonded N. (45)

Since the DNDs consist of a crystalline core and graphitic shell there is a possibility of $\mathrm{N}$ having different bonding states, one when it is within the crystalline core, and a second - when at the shell or/and surface region. In order to elucidate this, depth profiling via $\mathrm{Ar}^{+}$-sputtering was performed at the rate of $c a 0.2$ monolayer/min, and XPS spectra were acquired upon the incremental removal of every 0.5-0.6 monolayer (inset of the Fig. 4b). Upon the removal of $\sim 3$ monolayers of the graphitic shell a significant attenuation of the N2 peak was observed (Fig. 4b). The N1/N2 ratio increased $\sim 2$ times, indicating $\mathrm{N}-\mathrm{O}$ bonded species are located at the surface/surface region, whereas the $\operatorname{sp}^{\mathrm{x}}(\mathrm{x}=3$ and 2$)$ type $\mathrm{N}$ bonded to $\mathrm{C}$ is in the diamond core. Since the ratio changes in a linear manner (inset of Fig. 4b), one may conclude that $\mathrm{N}$ species are randomly distributed throughout the surface region. The corresponding C 1s peaks (along with the associated discussion) for the as-prepared and the sputtered samples are shown in the supplementary information (Fig. S4). 

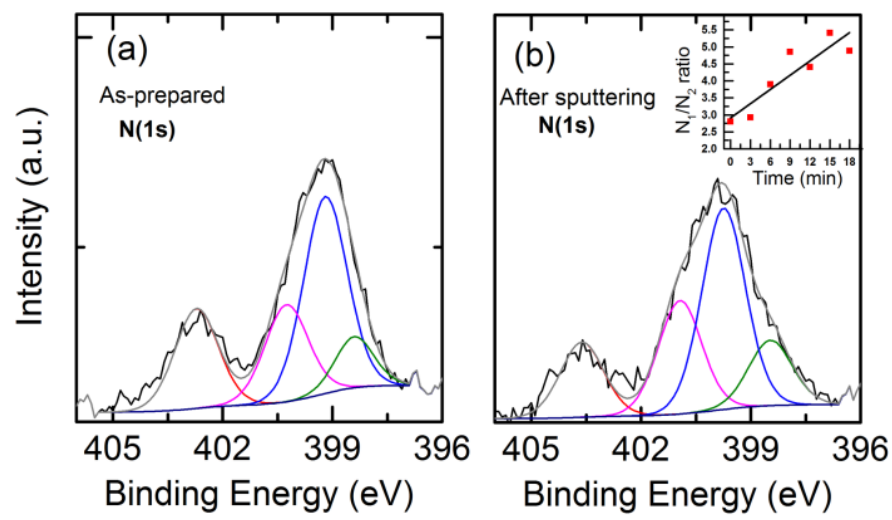

\section{Figure 4.}

XPS spectra of N1s for (a) as-prepared and (b) after $\mathrm{Ar}^{+}$-sputtering, exhibiting two pronounced spectral features. The inset in (b) shows a depth profiling via $\mathrm{Ar}^{+}$-sputtering performed at the rate of $c a 0.2$ monolayer/min. The spectra were acquired upon the incremental removal of every 0.5 0.6 monolayer.

The residual intensity of N2 peak (Fig. 4(b)) is attributed to the shadowing effect that might occur during depth profiling measurements of the powder samples. Unlike the single crystal or epitaxial-grown thin film surfaces that exhibit atomically flat planar morphology, the powder samples consist of supported individual nanoparticles and are highly corrugated, therefore, certain part of the surface is out of the 'line-of-sight' of the sputter gun, while still contributing to the photoemission signal, due to probing depth of XPS ( $c$ a $2 \mathrm{~nm}$ for the N1s). A significant peak shift towards higher binding energy is however observed after sputtering for the $\mathrm{N}-\mathrm{O}$ component initially at $402.5 \mathrm{eV}$ (by $\sim 0.8 \mathrm{eV}$ ) and the $\mathrm{sp}^{3}$ and $\mathrm{sp}^{2}$ - like 'N-C components' at $400.3 \mathrm{eV}$ and $399.2 \mathrm{eV}$, respectively (by $\sim 0.6 \mathrm{eV}$ ). The component initially at $398.4 \mathrm{eV}$ remains unchanged. 
The atomic concentrations of $\mathrm{C}, \mathrm{N}$ and $\mathrm{O}$ for the various sputtering cycles are shown Table SI3. It is found that as the $\mathrm{sp}_{2}$ shell is removed, the $\mathrm{N}_{\mathrm{sp}}{ }^{2}$ and $\mathrm{O}$ are reduced systematically (Table SI2). Since sputtering is a destructive technique per se, we switched to sequential heating of the as-prepared DNDs in order to be able to systematically control the removal $\mathrm{N}$ and $\mathrm{O}$ with $\mathrm{sp}^{2}$ shell. $\mathrm{Sp}^{2}$ shell was found to decrease systematically with $\mathrm{N}_{\mathrm{sp}}{ }^{2}$ and $\mathrm{O}$, however, around $650{ }^{\circ} \mathrm{C}$ the $\mathrm{sp}^{2}$ shell is again found to increase as a result of graphitization of the diamond core. Based on these experiments, we take the view that without physically damaging the DNDs, it is not possible to systematically control the $\mathbf{N}$ and $\mathrm{O}$ along with the $\mathrm{sp}^{2}$ content.

\section{Conclusions}

The presence of $\mathrm{N}$ in the sample is expected as it is the most common impurity in diamond, and is present in two distinct chemical states, $\mathrm{N}-\mathrm{O}$ bonded species distributed uniformly throughout the graphitic shell, and $\mathrm{C}-\mathrm{N} / \mathrm{C}=\mathrm{N}$ species in the diamond core. The combined luminescence and valence band study indicate that defect states, originating from the fullerenelike bound $\mathrm{C}$ species in the $\mathrm{sp}^{2}$ shells on the non-oxidized DNDs, serve as traps for the photoexcited charge carriers, in turn responsible for the quenching of the emission spectra. This work highlights the importance of the oxidation step in removing the graphitic shell and settles the longstanding doubt over the role of the shell in quenching the core-related PL activity, by suggesting the shell-related defect states to be acting as charge carrier traps.

\section{AUTHOR INFORMATION}

\section{Corresponding Author}

* Corresponding author: email: katsievk@SABIC.com 
The manuscript was written through contributions of all authors. All authors have given approval to the final version of the manuscript.

\section{Acknowledgement}

A.R.K., R.M., and K.K. would like to thank Prof. Osman M. Bakr of Solar and Photovoltaics Engineering Research Center (SPERC), KAUST for his continuous support and encouragement. Access to XPS and UPS at Nanoscale Characterization Facility of IU Nanoscience Center was provided by NSF Award DMR MRI-1126394.

\section{References}

1. Mochalin VN, Shenderova O, Ho D, Gogotsi Y. The properties and applications of nanodiamonds. Nat Nano. 2012;7(1):11-23; Williams OA. Nanodiamond. $\mathbf{1}^{\text {st }}$ ed. Royal Society of Chemistry; 2015, DOI: 10.1039/9781849737616

2. Artemov AS. Polishing nanodiamonds. Phys Solid State. 2004;46(4):687-95.

3. Dolmatov VY. Detonation nanodiamonds in oils and lubricants. J Superhard Mater. 2010;32(1):14-20.

4. Mochalin VN, Gogotsi Y. Wet Chemistry Route to Hydrophobic Blue Fluorescent Nanodiamond. Journal of the American Chemical Society. 2009;131(13):4594-5.

5. Chow EK, Zhang X-Q, Chen M, Lam R, Robinson E, Huang H, et al. Nanodiamond Therapeutic Delivery Agents Mediate Enhanced Chemoresistant Tumor Treatment. Science Translational Medicine. 2011;3(73):73ra21.

6. Chang Y-R, Lee H-Y, Chen K, Chang C-C, Tsai D-S, Fu C-C, et al. Mass production and dynamic imaging of fluorescent nanodiamonds. Nat Nano. 2008;3(5):284-8. 
7. Vaijayanthimala V, Cheng P-Y, Yeh S-H, Liu K-K, Hsiao C-H, Chao J-I, et al. The longterm stability and biocompatibility of fluorescent nanodiamond as an in vivo contrast agent. Biomaterials. 2012;33(31):7794-802.

8. Maze JR, Stanwix PL, Hodges JS, Hong S, Taylor JM, Cappellaro P, et al. Nanoscale magnetic sensing with an individual electronic spin in diamond. Nature. 2008;455(7213):644-7.

9. Burkat GK, Fujimura T, Dolmatov VY, Orlova EA, Veretennikova MV. Preparation of composite electrochemical nickel-diamond and iron-diamond coatings in the presence of detonation synthesis nanodiamonds. Diamond and Related Materials. 2005;14(11-12):17614.

10. Behler KD, Stravato A, Mochalin V, Korneva G, Yushin G, Gogotsi Y. NanodiamondPolymer Composite Fibers and Coatings. ACS Nano. 2009;3(2):363-9.

11. Neumann P, Mizuochi N, Rempp F, Hemmer P, Watanabe H, Yamasaki S, et al. Multipartite Entanglement Among Single Spins in Diamond. Science. 2008;320(5881):1326-9.

12. Manson NB, Harrison JP, Sellars MJ. Nitrogen-vacancy center in diamond: Model of the electronic structure and associated dynamics. Physical Review B. 2006;74(10):104303.

13. Kurtsiefer C, Mayer S, Zarda P, Weinfurter H. Stable Solid-State Source of Single Photons. Physical Review Letters. 2000;85(2):290-3.

14. Dwivedi N, Kumar S, Malik HK, Govind, Rauthan CMS, Panwar OS. Correlation of sp3 and sp2 fraction of carbon with electrical, optical and nano-mechanical properties of argondiluted diamond-like carbon films. Applied Surface Science. 2011;257(15):6804-10. 
15. Panda K, Sundaravel B, Panigrahi BK, Magudapathy P, Nandagopala Krishna D, Nair KGM, et al. Structural and electronic properties of nitrogen ion implanted ultra nanocrystalline diamond surfaces. Journal of Applied Physics. 2011;110(4):-.

16. Baranauskas V, Li BB, Peterlevitz A, Tosin MC, Durrant SF. Nitrogen-doped diamond films. Journal of Applied Physics. 1999;85(10):7455-8.

17. Tyler T, Shenderova O, Cunningham G, Walsh J, Drobnik J, McGuire G. Thermal transport properties of diamond-based nanofluids and nanocomposites. Diamond and Related Materials. 2006;15(11-12):2078-81.

18. Yeganeh M, Shahtahmasebi N, Kompany A, Goharshadi EK, Youssefi A, Šiller L. Volume fraction and temperature variations of the effective thermal conductivity of nanodiamond fluids in deionized water. International Journal of Heat and Mass Transfer. 2010;53(1516):3186-92.

19. Jelezko F, Wrachtrup J. Single defect centres in diamond: A review. physica status solidi (a). 2006;203(13):3207-25.

20. Tisler J, Balasubramanian G, Naydenov B, Kolesov R, Grotz B, Reuter R, et al. Fluorescence and Spin Properties of Defects in Single Digit Nanodiamonds. ACS Nano. 2009;3(7):195965.

21. Rabeau JR, Stacey A, Rabeau A, Prawer S, Jelezko F, Mirza I, et al. Single Nitrogen Vacancy Centers in Chemical Vapor Deposited Diamond Nanocrystals. Nano Letters. 2007;7(11):3433-7.

22. Naydenov B, Richter V, Beck J, Steiner M, Neumann P, Balasubramanian G, et al. Enhanced generation of single optically active spins in diamond by ion implantation. Applied Physics Letters. 2010;96(16):163108. 
23. Sjöström H, Stafström S, Boman M, Sundgren JE. Superhard and Elastic Carbon Nitride Thin Films Having Fullerenelike Microstructure. Physical Review Letters. 1995;75(7):13369.

24. Osswald S, Yushin G, Mochalin V, Kucheyev SO, Gogotsi Y. Control of sp2/sp3 Carbon Ratio and Surface Chemistry of Nanodiamond Powders by Selective Oxidation in Air. Journal of the American Chemical Society. 2006;128(35):11635-42.

25. Shenderova O, Koscheev A, Zaripov N, Petrov I, Skryabin Y, Detkov P, et al. Surface Chemistry and Properties of Ozone-Purified Detonation Nanodiamonds. The Journal of Physical Chemistry C. 2011;115(20):9827-37.

26. Peng W, Mahfouz R, Pan J, Hou Y, Beaujuge PM, Bakr OM. Gram-scale fractionation of nanodiamonds by density gradient ultracentrifugation. Nanoscale. 2013;5(11):5017-26.

27. Zhao FL, Gong Z, Liang SD, Xu NS, Deng SZ, Chen J, et al. Ultrafast optical emission of nanodiamond induced by laser excitation. Applied Physics Letters. 2004;85(6):914-6.

28. Chung PH, Perevedentseva E, Cheng CL. The particle size-dependent photoluminescence of nanodiamonds. Surface Science. 2007;601(18):3866-70.

29. Smith BR, Gruber D, Plakhotnik T. The effects of surface oxidation on luminescence of nano diamonds. Diamond and Related Materials. 2010;19(4):314-8.

30. Varun KAS, Andrei VZ, Ewa MG. Luminescent nanoparticles and their applications in the life sciences. Journal of Physics: Condensed Matter. 2013;25(19):194101.

31. Katsiev K, Ip AH, Fischer A, Tanabe I, Zhang X, Kirmani AR, et al. The Complete In-Gap Electronic Structure of Colloidal Quantum Dot Solids and Its Correlation with Electronic Transport and Photovoltaic Performance. Advanced Materials. 2014;26(6):937-42. 
32. Kirmani AR, Carey GH, Abdelsamie M, Yan B, Cha D, Rollny LR, et al. Effect of solvent environment on colloidal-quantum-dot solar-cell manufacturability and performance. Adv Mater. 2014;26(27):4717-23.

33. Santos MCd, Alvarez F. Structure and property relationships of amorphous CNx: a joint experimental and theoretical study. Brazilian Journal of Physics. 2000;30:495-507.

34. Díaz J, Paolicelli G, Ferrer S, Comin F. Separation of the sp3 and sp2 components in the C1s photoemission spectra of amorphous carbon films. Physical Review B. 1996;54(11):8064-9.

35. Hellgren N, Guo J, Luo Y, Såthe C, Agui A, Kashtanov S, et al. Electronic structure of carbon nitride thin films studied by X-ray spectroscopy techniques. Thin Solid Films. 2005;471(1-2):19-34.

36. Wesner D, Krummacher S, Carr R, Sham TK, Strongin M, Eberhardt W, et al. Synchrotronradiation studies of the transition of hydrogenated amorphous carbon to graphitic carbon. Physical Review B. 1983;28(4):2152-6.

37. Robertson J. Diamond-like amorphous carbon. Materials Science and Engineering: R: Reports. 2002;37(4-6):129-281.

38. Butenko YV, Krishnamurthy S, Chakraborty AK, Kuznetsov VL, Dhanak VR, Hunt MRC, et al. Photoemission study of onionlike carbons produced by annealing nanodiamonds. Physical Review B. 2005;71(7):075420.

39. Barinov A, Malcioğlu OB, Fabris S, Sun T, Gregoratti L, Dalmiglio M, et al. Initial Stages of Oxidation on Graphitic Surfaces: Photoemission Study and Density Functional Theory Calculations. The Journal of Physical Chemistry C. 2009;113(21):9009-13. 
40. Susi T, Kaukonen M, Havu P, Ljungberg MP, Ayala P, Kauppinen EI. Core level binding energies of functionalized and defective graphene. Beilstein Journal of Nanotechnology. $2014 ; 5: 121-32$.

41. Nirmal M, Brus L. Luminescence Photophysics in Semiconductor Nanocrystals. Accounts of Chemical Research. 1998;32(5):407-14.

42. Gao YK, Traeger F, Wöll C, Idriss H. Glycine adsorption and photo-reaction over ZnO(000ī) single crystal. Surface Science. 2014;624(0):112-7.

43. Turner S, Shenderova O, Da P F, Lu Y, Yücelen E, Verbeeck J, Lamoen D, Van T G. Aberration-corrected microscopy and spectroscopy analysis of pristine, nitrogen containing detonation nanodiamond. Physica Status Solidi (A). 2013; 210(10):1976-84.

44. Kennou S, Logothetidis S, Sygellou L, Laskarakis A, Sotiropoulou D, Panayiotatos Y. Variation of nitrogen incorporation and bonding configuration of carbon nitride films studied by X-ray photoelectron spectroscopy (XPS) and Fourier transform infrared (FT-IR) spectroscopic ellipsometry. Diamond and Related Materials. 2002;11(3-6):1183-7.

45. Zhu Y, Lin Y, Zhang B, Rong J, Zong B, Su DS. Nitrogen-Doped Annealed Nanodiamonds with Varied sp2/sp3 Ratio as Metal-Free Electrocatalyst for the Oxygen Reduction Reaction. ChemCatChem. 2015, DOI: 10.1002/cctc.201402930.

46. Shenderova O, Hens S, McGuire G. Seeding slurries based on detonation nanodiamond in DMSO. Diamond and Related Materials. 2010;19(2-3):260-7. 